\title{
Telangiectasia hemorrágica hereditaria: \\ Tratamiento farmacológico
}

\author{
Sonia Molinos-Castro, Paula María Pesqueira-Fontán, \\ José Antonio Díaz-Peromingo
}

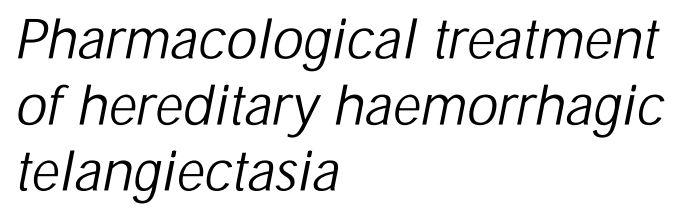

Hereditary haemorrhagic telangiectasia (HHT) of Rendu-OslerWeber disease is a multisystem vascular dysplasia characterized by the presence of multiple telangiectasia, mainly affecting mucosal tissue and arterial-venous connections. It commonly affects lungs, liver and central nervous system. Bleeding is the most common symptom and its treatment can be surgical or pharmacological. We herein review the systemic therapy that attempts to minimize bleeding as well as blood transfusion therapy. Blood therapy and fibrinolytic treatment especially aminocaproic acid and tranexamic acid are discussed. Danazol, hormone therapy, and other less common drugs used in the treatment of HHT are also reviewed (Rev Méd Chile 2009; 137: 695-700).

(Key words: Blood transfusions; Fibrinolysis; Telangiectasia, hereditary hemorrhagic).

Recibido el 6 de marzo, 2008, Aceptado el 9 de septiembre, 2008.

Servicio de Medicina Interna. Hospital da Barbanza. Riveira. A Coruña.

L

a telangiectasia hemorrágica hereditaria $\boldsymbol{\omega}$ (THH), también conocida como enfermedad de Rendu-Osler-Weber fue descrita inicialmente por Sutton en $1864^{1}$, pero fue Rendu en 1896 el primero en reconocerla como una entidad nosológica propia ${ }^{2}$. Las primeras series con agrupaciones de casos fueron realizadas por Osler en $1901^{3} \mathrm{y}$ Weber en $1907^{4}$ pero el nombre actual de THH se lo dio Hanes en $1909^{5}$. La THH es una displasia vascular multisistémica con un patrón de herencia autonómico dominante y una prevalencia estima-

$\overline{\text { Correspondencia a: Dr. José Antonio Díaz Peromingo. C/ Doc- }}$ tor Teijeiro 29, 5․ 15701. Santiago de Compostela. A Coruña. E- mail: jadiazperomingo@hotmail.com; jose.antonio.diaz.peromingo@sergas.es da probablemente superior a $1 / 10.000$ con una distribución geográfica muy amplia ${ }^{6}$. Este desorden genéticamente heterogéneo se encuentra ligado a dos loci: el primero es el HHT1 y es causado por mutaciones en el gen de la endoglina (ENG) que se localiza en el brazo largo del cromosoma 97 y el segundo es el HHT2, que está causado por mutaciones en el gen del receptor de actividad tipo cinasa 1 (ALK1) que está localizado en el brazo largo del cromosoma 128. Existe un tercer subtipo, más raro, que se asocia con la poliposis colónica juvenil y está causado por mutación en el SMAD- $4^{9}$. Ambos genes (ENG y ALK1) codifican para proteínas receptoras del factor de crecimiento transformante $\beta$ (TGFß) expresado por las células endoteliales siendo la endoglina un receptor accesorio y ALK-1 un receptor de tipo I. Esto 
implica una regulación positiva de la angiogéne$\operatorname{sis}^{10}$.

La enfermedad se caracteriza por un lado por presentar telangiectasias, sobre todo afectando a las mucosas oral, nasal y gastrointestinal y por otro, por la presencia de malformaciones arteriovenosas o conexiones directas arteria-vena que afectan sobre todo a pulmones, hígado y sistema nervioso central. Ambas lesiones predisponen a eventos hemorrágicos. Dada la pluralidad de órganos que se pueden afectar en esta enfermedad, el enfoque debe ser multidisciplinario y en el tratamiento se usan técnicas de embolización, resección quirúrgica o estereotáxica, laserterapia, inyección de fármacos en las telangiectasias 0 malformaciones arteriovenosas 0 , en casos de afectación hepática, el trasplante hepático ${ }^{11}$. En ocasiones el sangrado debe ser tratado con terapia transfusional o fármacos que disminuyan dicho sangrado o lo prevengan.

El tratamiento médico de la THH está todavía lejos de su estandarización. El presente artículo pretende revisar los fármacos empleados a nivel sistémico para prevenir el sangrado en pacientes con THH y la terapia transfusional empleada. En primer lugar se revisa el tratamiento con hemoderivados e hierro. Posteriormente se analiza el papel de los antifibrinolíticos, en especial el ácido tranexámico y aminocaproico, y otros fármacos como el danazol y la terapia hormonal con estrógenos y progestágenos. En un último término, se revisan otros fármacos menos comunes, pero que también se han usado en ocasiones con éxito en el tratamiento de la $\mathrm{THH}$.

1. Hierro y hemoderivados. Debido a los frecuentes sangrados que pueden originarse por las lesiones vasculares en distintas localizaciones (epístaxis, sangrado gastrointestinal, pulmonar, etc.) es frecuente la existencia de anemia ferropénica en la mayoría de los pacientes con $\mathrm{THH}^{12}$. El tratamiento de elección siempre es el hierro oral si es posible, se prefieren las sales ferrosas por su mejor absorción gastrointestinal. Otras opciones son la administración parenteral de hierro (preferentemente por vía intravenosa) que debe restringirse a casos excepcionales como malabsorción, intolerancia al hierro oral o anemias severas dado el riesgo de reacciones adversas graves. La transfusión de hemoderivados se reserva para situacio- nes más graves que requieran una rápida corrección de la anemia existente.

2. Terapia antifibrinolítica. Uno de los factores implicados en la patogenia de este síndrome es la alteración en la agregación y adhesión plaquetarias. En este sentido se ha descrito una disfunción plaquetaria evidenciada como una defectuosa agregación plaquetaria con ADP (adenosín-difosfato) o colágeno-adrenalina y defectuosa retracción del coágulo sanguíneo. Estos defectos plaquetarios contribuirían al deterioro de la hemostasia primaria pudiendo contribuir a una mayor severidad en el sangrado ${ }^{13-16}$. El ácido aminocaproico es un potente antifibrinolítico que inhibe los activadores del plasminógeno y estabiliza la formación del coágulo en la pared del vaso telangiectásico haciendo posible los depósitos de fibrina ${ }^{17}$. Hay diversos estudios que han demostrado el aumento de concentración de la actividad del activador del plasminógeno en la pared de estos vasos ${ }^{18-21}$. Han sido muchos los ensayos realizados sobre la capacidad de reducir la frecuencia de epistaxis en estos pacientes con ácido aminocaproico con muy diferentes resultados, lo que nos puede hacer pensar que hay además otros factores implicados en la patogenia de esta enfermedad. Lo que sí puede deducirse de estos estudios es que, en un alto porcentaje de casos, estos tratamientos reducen la frecuencia y severidad de las epistaxis logrando un mejor control de las cifras de hemoglobina y disminuyendo las necesidades transfusionales ${ }^{21,22}$.

El otro fármaco antifibrinolítico empleado es el ácido tranexámico que tiene una acción prolongada y ha demostrado beneficios en el tratamiento de epistaxis en cuanto a frecuencia y severidad de los síntomas, en pacientes seleccionados ${ }^{23}$. Este fármaco estimula la expresión de ALK-1 y endoglina, así como la actividad de ALK-1/endoglina. Hay diferentes trabajos que sugieren que su administración diaria a elevadas dosis que varían entre los $2 \mathrm{~g}$ y los $6 \mathrm{~g}$, disminuye los episodios de sangrado en los pacientes afectos sin acompañarse de efectos secundarios significativos ${ }^{24}$.

En ambos casos se han realizado muy diversos $\mathrm{y}$ variados estudios recogiendo la frecuencia y severidad de los sangrados, así como la cifra de hemoglobina y la necesidad de tratamiento transfusional. Los resultados han sido dispares y en 
ocasiones contradictorios, lo que pone en evidencia que no hay una terapia claramente efectiva para el tratamiento de esta patología y que en aquellos pacientes en los que los síntomas son muy severos, se debería plantear la posibilidad de combinar estos tratamientos con otras terapias más agresivas ${ }^{25}$.

Un efecto secundario importante a tener en cuenta en pacientes que reciben tratamiento antifibrinolítico es la trombosis. Teóricamente, en pacientes con $\mathrm{THH}$, la actividad fibrinolítica está localmente aumentada en las lesiones haciendo más fácil la hemorragia. Estímulos que inician la coagulación también inician la generación de plasmina que actúa sobre la fibrina dando lugar a la formación de productos de degradación de la misma. Tanto el ácido aminocaproico como el tranexámico, al ser análogos de la lisina, inhiben la unión de la plasmina a la fibrina ${ }^{26}$. Así, la hemostasia final es fruto de un adecuado balance entre trombosis y fibrinolisis. De este modo, la actividad fibrinolítica local aumentada lleva a la existencia de concentraciones plasmáticas elevadas de dímero $\mathrm{D}$ sin que exista una concomitante coagulopatía de consumo ${ }^{27}$ y puede ser debida tanto a la existencia de un endotelio alterado ${ }^{21} 0$ a una mala respuesta fisiológica a la formación de trombos dentro de las lesiones. La existencia de concentraciones elevadas de antígeno del factor VIII de la coagulación también se ha relacionado con la posibilidad de aparición de enfermedad tromboembólica venosa en estos pacientes ${ }^{28}$. De forma infrecuente, ambos fármacos pueden producir hipotensión, el ácido tranexámico alteraciones retinianas y el ácido aminocaproico rabdomiolisis ${ }^{26}$.

3. Terapia hormonal. Existen varios estudios no randomizados en los que se ha demostrado la utilidad de la terapia hormonal en pacientes con sangrados recurrentes por malformaciones arteriovenosas (MAV) gastrointestinales y nasofaríngeas, ya sea en el seno de THH o no. Dentro de estos tratamientos hormonales se encuentran los preparados con estrógenos, el tamoxifeno, el danazol, el octreótido y la desmopresina ${ }^{29,30}$.

En lo que se refiere a los estrógenos y progestágenos, la administración de estrógenos (ya sean sistémicos o tópicos) produce una metaplasia escamosa del epitelio que disminuye la fragilidad de las mucosas, mediada por la unión del estrógeno al receptor tipo $B$ estrogénico presente en la mucosa de pacientes con $\mathrm{THH}^{31}$. El estriol (un metabolito de acción débil del estradiol) aplicado de forma tópica y oleosa al $0,1 \%$ sobre la mucosa nasal durante periodos superiores a 12 meses disminuye la incidencia de epístaxis en pacientes con $\mathrm{THH}^{32-}$ 34. La administración de $30-50 \mathrm{mcg}$ de etinilestradiol junto con 1-1,5 mg de nortestosterona al día en pacientes con sangrado gastrointestinal frecuente $\mathrm{e}$ importante por MAV ha demostrado una disminución significativa de los requerimientos transfusionales $^{35,36}$. También se han utilizado altas dosis de estrógenos conjugados (equivalente a $625 \mathrm{mcg} /$ día de etinilestradiol) con éxito, aunque no hay estudios randomizados que lo respalden $\mathrm{y}$, por otro lado, otros estudios no encuentran beneficio en la administración de esta terapia hormonal ${ }^{37}$.

La indicación de tratamiento hormonal puede diferir ligeramente en el caso de que el paciente sea un hombre o una mujer. En este sentido, las mujeres tienen mayor riesgo de desarrollar malformaciones arteriovenosas pulmonares y probablemente enfermedad hepática y hemorragia cerebral que los hombres ${ }^{38-40}$. Esto puede ser debido a una acción directa sobre la vasculatura telangiectásica por parte de las hormonas sexuales femeninas, sustentado esto en varios estudios que demuestran tratamiento exitoso de episodios de sangrado fundamentalmente gastrointestinal con estrógenos-porgestágenos ${ }^{35,29,41}$ y en el hallazgo de receptores de progesterona en los vasos de pacientes con $\mathrm{THH}^{42}$. También se ha puesto en relación esta diferencia entre sexos con cambios hemodinámicos que se pudieran producir durante el embarazo en mujeres con $\mathrm{THH}^{43}$. De acuendo con esto el uso sistémico de combinaciones de estrógenos y progestágenos a dosis habituales contraceptivas parece una opción razonable para disminuir los episodios de sangrado secundario a THH en mujeres fértiles ${ }^{44}$.

El tamoxifeno es un fármaco antineoplásico hormonal antiestrogénico (antagonista del receptor) cuyo mecanismo de acción es similar a los estrógenos y con muy buena tolerancia. Ha demostrado en algunos casos poder disminuir el sangrado nasal en pacientes postmenopáusicas con THH y parece una buena opción en mujeres postmenopáusicas donde el tratamiento con estrógenos tendría más potencialidad de efectos secundarios indeseables ${ }^{44}$. 
El danazol es un derivado androgénico de acción débil con acción antiestrogénica y antiprogesterona. Su papel en THH es controvertido y hoy está en desuso ${ }^{12,45}$.

La somaostatina, el octreótido (análogo de la somatostatina) y la desmopresina (análogo de la vasopresina) son hormonas que se utilizan en hemorragias digestivas, ya que producen vasoconstricción a nivel esplácnico, pero no hay estudios específicos en pacientes con $\mathrm{THH}^{46}$.

4. Otros tratamientos. Ocasionalmente se han comunicado casos puntuales de utilización de distintos tratamientos en la THH como el bosentan, la talidomida, el sirolimus y el interferón. El bosentan es un antagonista del receptor de la endotelina-1 que, debido a su efecto reductor de la hipertensión pulmonar se ha usado en el tratamiento de pacientes con hipertensión pulmonar asociada a $\mathrm{THH}^{47}$. El sirolimus es un agente inmunosupersor usado sobre todo en el trasplante renal. Se ha descrito su utilidad en una paciente con THH que recibió un trasplante hepático en la que desaparecieron las telangiectasias cutáneas, gastrointestinales y de mucosa oral que presentaba la paciente. Se ha postulado que podría deberse a un efecto del sirolimus sobre el factor de crecimiento del endotelio vascular ${ }^{48}$. La talidomida ha sido también utilizada con efectividad para el tratamiento del sangrado gastrointestinal de un paciente con $\mathrm{THH}$ asociada a mieloma múltiple con cese de los episodios hemorrági$\cos ^{49}$. Por último, se ha descrito también la probable efectividad del interferón alfa tanto en pacientes oncológicos ${ }^{50}$ como en los que lo

\section{REFERENCIAS}

1. Sutton HG. Epistaxis as an indication of impaired nutrition, and of degeneration of the vascular system. Medical Mirror 1864; 1: 769.

2. RENDU H. Epistaxis repetees chez un sujet porteus de petits angiomes cutanes et muquez. Gaz des Hôpitaux Civils et Militaires 1896; 135: 1322-3.

3. OSLER W. On a family form of recurring epistaxis, associated with multiple telangiectases of the skin and mucous membranes. Bull Johns Hopkins Hosp 1901; 7: 333-7.

4. WEBER FP. Multiple hereditary developmental angiomata (telangiectases) of the skin and mucous mem- recibían para tratamiento de una infección por el virus de la hepatitis $\mathrm{C}^{51}$ con $\mathrm{THH}$ asociada.

\section{CONCLUSIONES}

La ausencia de ensayos randomizados y controlados no permite obtener unas recomendaciones formales sobre el tratamiento médico de estos pacientes. La terapia férrica con hierro oral o más raramente parenteral es el pilar fundamental del control de la anemia en estos pacientes. La terapia transfusional debe dejarse para casos excepcionales de inestabilidad hemodinámica o necesidades perentorias de recuperación de la volemia sanguínea. En el momento actual, el tratamiento con fármacos antifibrinolíticos como el ácido tranexámico o aminocaproico es el más comúnmente usado y del que se dispone de mayor soporte documental, sin embargo, no existe unanimidad sobre su eficacia en todos los pacientes con THH. La terapia hormonal también ha mostrado resultados dispares en diversos estudios realizados como se ha visto previamente. Otros tratamientos, en particular el bosentan y el sirolimus parecen prometedores de cara al futuro, pero se necesitan ensayos clínicos amplios y randomizados para poder recomendar de forma extensa su uso. En resumen, el tratamiento farmacológico de las complicaciones hemorrágicas de los pacientes con THH dista mucho de estar claro y ser óptimo y la complejidad en ocasiones de su manejo hace necesaria la cooperación interdisciplinar para lograr los mejores objetivos de tratamiento en estos pacientes.

branes associted with recurring haemorrhages. Lancet 1907; 2: 160-2.

5. HANES FM. Multiple hereditary telangiectases causes hemorrhage (hereditary hemorrhagic telangiectasia). Bull Johns Hopkins Hosp 1909; 20: 63-73.

6. Pérez Del Molino A, Zarrabeitía $R$, Fernández A. Telangiectasia hemorrágica hereditaria. Med Clin (Barc) 2005; 124: 583-7.

7. McDonald MT, Papenberg KA, Ghosh S, Glatfelter AA, Biesecker BB, Helmbold EA et al. A disease locus for hereditary haemorrhagic telangiectasia maps to chromosome 9q33-34. Nat Genet 1994; 6: 197-204.

8. Johnson DW, Berg JN, Gawone CJ, MCALUSTer KA, WARNER JP, HeLmBold EA ET AL. A second locus for 
hereditary hemorrhagic telangiectasia maps to chromosome 12. Genome Res 1995; 5: 21-8.

9. Abadía SA, Letarte M. Hereditary haemorrhagic telangiectasia: current views on genetics and mechanisms of disease. J Med Genet 2006; 43: 97.

10. Goumans MJ, Valdimarsdottir $G$, Itoh S, Rosendahl A, Sideras P, Ten DijKe P. Balancing the activation state of the endothelium via two distinct TGF-b type I receptors. EMBO J 2002; 21: 1743-53.

11. McDonald J, Bayrak-Toydemir P. Hereditary hemorrhagic telangiectasia. Haematologica 2005; 90: 728-32.

12. Longacre AV, Gross CP, Gaшtelu M, Henderson KJ, White RI JR, Proctor DD. Diagnosis and management of gastrointestinal bleeding in patients with hereditary hemorrhagic telangiectasia. Am J Gastroenterol 2003; 98: 59-65.

13. LaRSSON SO. Osler's disease with impaired adhesion and aggregation of platelets. Acta Med Scand 1974; 196: 133-6.

14. PANDOlFI M, Ehinger B. Conjunctival bleeding in Osler's disease with associated platelet dysfunction: a case report. Acta Ophthalmol (Copenh) 1978; 56: 75-82.

15. MuCKLE TJ. Low in-vivo adhesive-platelet count in hereditary haemorrhagic telangiectasia. Lancet 1964; 2: 880-2.

16. Endo Y, Mamiya S, NitTsu H, Miura A, Nashimura S. Reduced platelet aggregation induced by epinephrine and idiopathic thrombocytopenic purpura in hereditary haemorrhagic telangiectasia. Thromb Haemost 1984; 52: 369.

17. ANNICHINo-BizZACCHI JM, FACChini RM, TORRESAN MZ, ARRUDA VR. Hereditary hemorrhagic telangiectasia response to aminocaproic acid treatment. Thromb Res 1999; 96: 73-6.

18. Peschen M, Rogers AA, Chen WY, Vanscheidt W. Modulation of urokinase-type and tissue-type plasminogen activator occurs at an early stage of progressing stages of chronic venous insufficiency. Acta Derm Venereol 2000; 80: 162-6.

19. Он SP, Seki T, Goss KA, Imamura T, Yi Y, Donahoe PK ET AL. Activin receptor-like kinase 1 modulates transforming growth factor-beta 1 signaling in the regulation of angiogenesis. Proc Acad Sci USA 2000; 97: 2626-31.

20. Guerrero-Esteo M, Lastres P, Letamendia A, PérezAlvarez MJ, Langa C, López LA et aL. Endoglin overexpression modulates cellular morphology, migration, and adhesión of mouse fibroblasts. Eur J Cell Biol 1999; 78: 614-23.

21. KwaAn HC, Silverman S. Fibrinolytic activity in lesions of hereditary haemorrhagic telangiectasia. Arch Dermatol 1973; 107: 571-3.

22. Fiorelia ML, Ross DA, White RI, Sabba C, Fiorelia R. Hereditary haemorrhagic telangiectasia: state of the art. Acta Otorhinolaryngol Ital 2004; 24: 330-6.
23. Sabba C, Galiteli M, Palasciano G. Efficacy of unusually high doses of Tranexamic Acid for the treatment of epistaxis in Hereditary Hemorrhagic Telangiectasia. N Engl J Med 2001; 345: 926.

24. Fernández A, Garrido EM, Sanz F, Ramírez JR, Morales C, ZaRrabetTía R ET AL. Terapeutic action of tranexamic acid in hereditary haemorrhagic telangiectasia: regulation of ALK-1/endoglin pathway in endothelial cells. Thromb Haemost 2007; 97: 254-62.

25. Sabba C, Pascum G, Cirum A, Galutelu M, Virgilio G, Guastamacchia E et al. Rendu-Osler-Weber disease: experience with 56 patients. Ann Ital Med Int 2002; 17: 173-9.

26. PHiLss MD. Stopping bleeding in hereditary telagiectasia. N Engl J Med 1994; 330: 1822-3.

27. Sureda A, Cesar J, García-Frade LJ, García-Avello A, FERNÁNDEZ-FuERTES I, NAVARRO JL. Hereditary haemorrhagic telangiectasia: analysis of platelet aggregation and fibrinolytic system in seven patients. Acta Haematol 1991; 85: 119-23.

28. Shovlin CL, Sulaiman NL, Govani FS, Jackson JE, BEgBIE ME. Elevated factor VIII in hereditary haemorrhagic telangiectasia (HHT): association with venous thromboembolism. Thromb Haemost 2007; 98: 1031-9.

29. Marshall JK, Hunt RH. Hormonal therapy for bleeding gastrointestinal mucosal vascular abnormalities: a promising alternative. Eur J Gastroenterol Hepatol 1997; 9: 521-5.

30. Bronner MH, Pate MB, Cunningham JT, Marsh WH. Estrogen-progesterone therapy for bleeding gastrointestinal telangiectasias in chronic renal failure. An uncontrolled trial. Ann Intern Med 1986; 105: 371-4.

31. Pau H, Carney AS, Walker R, Murty GE. Is oestrogen therapy justified in the treatment of hereditary haemorrhagic telangiectasia: a biochemical evaluation. Clin Otolaryngol Allied Sci 2000; 25: 547-50.

32. Sadick H, Bergler WF, Oulmi-Kagermann J, Naim R, SAdick M, HormanN K ET AL. Estriol induced squamous metaplasia on the nasal mucosa in patients with hereditary hemorrhagic telangiectasia. Arch Med Res 2005; 36: 468-73.

33. Sadick H, Naim R, Oulmi J, Horman K, Bergler W. Plasma surgery and topical estriol: effects on the nasal mucosa and long-term results in patients with Osler's disease. Otolaryngol Head Neck Surg 2003; 129: 233-8.

34. Bergler W, Sadick H, Gotte K, Riedel F, Hormann K. Topical estrogens combined with argon plasma coagulation in the management of epistaxis in hereditary hemorrhagic telangiectasia. Ann Otol Rhinol Laryngol 2002; 111: 222-8.

35. Van Cutsem E, Rutgeerts P, Vantrappen G. Treatment of bleeding gastrointestinal vascular malformations with oestrogen-progesterone. Lancet 1990; 335: 953-5. 
36. Moss SF, Ghosh P, Thomas DM, Jackson JE, Calam J. Gastric antral vascular ectasia: maintenance treatment with oestrogen-progesterone. Gut 1992; 33: 715-7.

37. Lewis BS, Salomon P, Rivera-MacMurray S, Kornbluth AA, Wenger J, WAYE JD. Does hormonal therapy have any benefit for bleeding angiodysplasia? J Clin Gastroenterol 1992; 15: 99-103.

38. White RI Jr, Lynch-Nyhan A, Terry P, Buescher PC, FARMLETT EJ, CharNas L ET AL. Pulmonary arteriovenous malformations: techniques and long-term outcome of embolotherapy. Radiology 1988; 169: 663-9.

39. MARTINI GA. The liver in hereditary haemorrhagic teleangiectasia: an inborn error of vascular structure with multiple manifestations: a reappraisal. Gut 1978; 19: 531-7.

40. Graf CJ, Perret GE, Torner JC. Bleeding from cerebral arteriovenous malformations as part of their natural history. J Neurosurg 1983; 58: 331-7.

41. SHOvLin CL, LETARTE M. Hereditary haemorrhagic telangiectasia and pulmonary arteriovenous malformations: issues in clinical management and review of pathogenic mechanisms. Thorax 1999; 54: 714-29.

42. Richtsmeier W, Weaver G, Streck W, Jacobson H, DEWELL R, OLSON J. Estrogen and progesterone receptors in hereditary hemorrhagic telangiectasia. Otolaryngol Head Neck Surg 1984; 92: 564-70.

43. Shovin CL, Winstock AR, Peters AM, Jackson JE, Hughes JM. Medical complications of pregnancy in hereditary haemorrhagic telangiectasia. QJM 1995; 88: 879-87.
44. JAMESON JJ, CAVE DR. Hormonal and antihormonal therapy for epistaxis in hereditary hemorrhagic telangiectasia. Laryngoscope 2004; 114: 705-9.

45. Staffa F, Bartone M, Mancuso G, Mattiol PL, Pujia A, Gnasso A. Malattia di Rendu-Osler-Weber. Descrizione di un caso clinico. Minerva Med 1996; 87: 471-4.

46. LEwIS BS. Medical and hormonal therapy in occult gastrointestinal bleeding. Semin Gastrointest Dis 1999; 10: 71-7.

47. Bonderman D, Nowotny R, Skoro-Sajer N, Adibrecht C, LANG IM. Bosentan therapy for pulmonary arterial hypertensión associated with hereditary haemorrhagic telangiectasia. Eur J Clin Invest 2006; 36 Suppl 3: 71-2.

48. Skaro AI, Marotta PJ, Mcalister VC. Regession of cutaneous and gastrointestinal telangiectasia with sirolimus and aspirin in a patient with hereditary haemorrhagic telangiestasia. Ann Intern Med 2006; 144: 226.

49. Pérez-Encinas M, Rabuñal-Martínez MJ, BeLo-López JL. It's the thalidomide effective for the treatment of gastrointestinal bleeding in Hereditary Hemorrhagic Telangiectasia? Haematologica 2002; 87: 34.

50. Wheatley-Price P, Shovlin C, Chao D. Interferon for metastatic renal cell cancer causing regression of hereditary hemorrhagic telangiectasia. J Clin Gastroenterol 2005; 39: 344-5.

51. Massould OI, Youseff WI, Mulen KD. Resolution of hereditary hemorrhagic telangiectasia and anemia with prolonged alpha-interferon therapy for chronic hepatitis C. J Clin Gastroenetrol 2004; 38: 377-9. 